\title{
A Possible Explanation for the Different Mean Speeds of Halo and Limb CMEs
}

\author{
J.X. Cheng, C.T. Yeh, D.M. Ding and P.F. Chen \\ Department of Astronomy, Nanjing University, Nanjing, 210093, China \\ email:chengjianxia@nju.org.cn
}

\begin{abstract}
In order to explain the different average speeds between halo and limb CMEs, we investigate the relationship between the brightness and speed for 17 halo CMEs. It is found that faster CMEs tend to be brighter, which implies that many halo CMEs with slow speeds are missed in observation owing to the limited sensitivity of LASCO detectors or identifications. As a result, the statistical average speed of halo CMEs turns to be much larger than that of limb CMEs.
\end{abstract}

Keywords. Sun: coronal mass ejections (CMEs)

\section{Introduction}

Coronal mass ejections are the most significant eruptive events in the solar atmosphere. In most cases, CMEs have an angular width of tens of degrees; however, CMEs appears to be fully or partially halo-like when their source regions close to the solar disk center. In this sense, CMEs can be categorized into limb and halo events. According to statistics, the average speed of halo CMEs is much higher than that of limb events (Webb et al. 1999; Michalek et al. 2003). It is generally assumed that halo CMEs are more energetic than limb events in order to explain the speed difference. However, such an explanation is not so justified since the occurrence rate should be the same for all longitudes. In this paper, we suppose that such a speed difference is possible due to an observation effect, as proposed by Andrews (2002).

\section{Data analysis}

We select 17 halo CMEs that are associated with flares for a statistical study. The brightness is derived by integrating over the whole area of each CME. For simplicity, the onset time of the CME is roughly considered as the peak time of the associated flare. The arrival time is determined by GOES observations as the time when the front loop propagates to the GOES satellite.

\section{Our result}

As shown in Fig. 1, the brightness and speed of CMEs are positively correlated with a correlation coefficient of 0.73 . The faster the CME is, the larger the brightness is. This implies that faster CMEs are brighter that can be more easily observed while slower ones are less bright that are more difficult to observe. Since the emission of CMEs is from the Thomson scattering that peaks at a direction perpendicular to the ray path of photons from the photosphere, limb CMEs are easier to detect than halo CMEs, especially for those CMEs with a lower speed and brightness. Therefore, some slow halo CMEs are missed in observations for the limited sensitivity of the instrument while they 


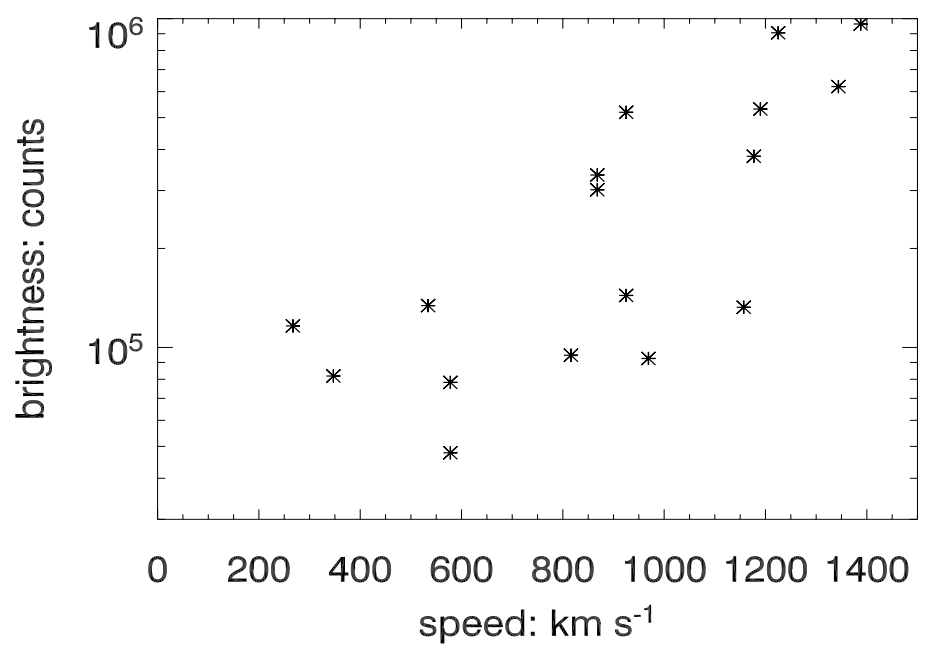

Figure 1. Brightness vs speed of CMEs showing a positive correlation. The faster the CME is, the larger the brightness is.

can possible be detected if appearing at the limb; as a result, the average speed of halo CMEs is larger than limb ones.

\section{Conclusion}

We investigate the relationship between the brightness and speed using a sample of 17 halo CMEs. It is found that faster CMEs are brighter. This can be understood as a pileup effect: CMEs with a larger speed can accumulate more coronal plasma during their propagation, and hence are brighter considering the effect of the Thomson scattering. Since the Thomson scattering is most significant when the CMEs propagate in the plane of the sky, halo CMEs are more difficult to detect than limb CMEs, especially for those CMEs with a slow speed. In other words, only fast halo CMEs can be easily detected while quite a number of slow halo CMEs are missed by the SOHO/LASCO coronagraph as their brightness is below the detection sensitivity. As a result, the average speed of halo CMEs is apparently larger than that of limb CMEs. Our result confirms the proposal by Andrews (2002).

\section{Acknowledgements}

We are grateful to Dr. K. Dere and A. Vourlidas for their useful comments. SOHO is a project of international cooperation between ESA and NASA. This work is supported by FANEDD (200226), NSFC (49990451, 10221001, 10333040,10403003) and NKBRSF (G20000784).

\section{References}

Andrews, M. D. 2002, Solar Phys. 208, 317

Brueckner, G.E., Howard, R.A., Koomen, M.J., et al. 1995, Solar Phys. 162, 357

Michalek, G., Gopalswamy, N., \& Yashiro, S. 2003, ApJ 584, 472

Webb, D.F., St. Cyr, O.C., Plunkett, S.P., Howard, R.A., \& Thompson, B.J. 1999, BAAS 31, 853 